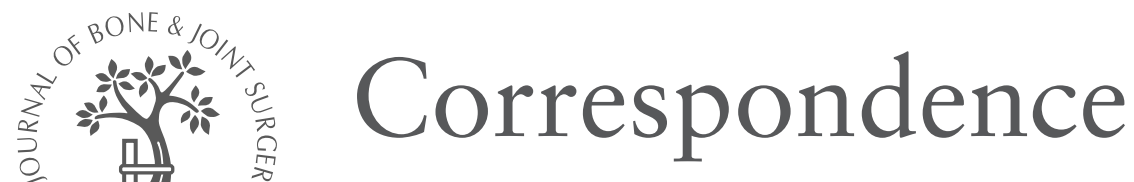

We welcome letters to the Editor concerning articles which have recently been published. Such letters will be subject to the usual stages of selection and editing; where appropriate the authors of the original article will be offered the opportunity to reply.

Letters should normally be under $\mathbf{3 0 0}$ words in length, doublespaced throughout, signed by all authors and fully referenced. The edited version will be returned for approval before publication.

C2005 British Editorial Society of Bone and Joint Surgery doi:10.1302/0301-620X.87B4.16232

$\$ 2.00 \mathrm{~J}$ Bone Joint Surg [Br] 2005;87-B:588-90.

\section{Infection after total hip arthroplasty}

Sir,

We read with interest the paper in the September 2003 issue by Blom et al ${ }^{1}$ entitled 'Infection after total hip arthroplasty'. The definition of infection is clearly essential when comparing infection rates. All authors agreed that superficial infection is far more common than deep infection, and there are many patients who have an inflamed wound who are given antibiotics either whilst in hospital or by the GP with no bacterial proof of infection. It would appear from their results, there were only seven of 1567 patients who had proven deep infection, an infection rate of $0.45 \%$.

The Royal Orthopaedic Hospital in Birmingham has taken a very different approach to investigating the incidence of infection following hip replacement. For the past 16 years, the Control of Infection Committee has monitored all positive cultures obtained from any patient who has previously undergone a total hip or knee replacement at this hospital. All cases where an organism has been cultured are investigated, and the infection labelled as either superficial or deep. If a superficial infection subsequently turns into a deep infection then the patient is reclassified, although the risk of this has been found to be low. ${ }^{2}$ The hospital has information on 6842 primary total hip replacements in whom there is a $0.4 \%$ deep infection rate and a $1.7 \%$ superficial infection rate. For the 5863 total knee replacements the deep infection rate is $0.5 \%$ and superficial infection rate is $1.8 \%$.

Both of these results appear to be better than those reported in the Trent Arthroplasty Register, but are more in keeping with those reported from other large series. ${ }^{3,4}$

We believe that neither our nor Blom's method of collecting data is perfect. Blom et $\mathrm{al}^{1}$ have relied upon patients' recollections, which one would hope would be fairly accurate, while we have relied upon hospital records and, therefore, may have missed patients who have been treated elsewhere for post-operative infection.

With the mandatory requirements coming into force in the near future for reporting the site of surgical infection, it is essential that we all agree on what data should be collected and how they should be collected. We are currently carrying out a retrospective review comparing the results from a two-year period, using the method of Blom et $\mathrm{al}^{1}$ to see how many patients with deep infection have been missed by our method of data collection, but ultimately it is likely that no one mechanism can be foolproof.

R. J. GRIMER, FRCS(Ed) (Orth)

A. ABUDU, FRCS(Orth)

The Royal Orthopaedic Hospital, Birmingham, UK.

1. Blom AW, Taylor AH, Pattison G, Whitehouse S, Bannister GC. Infection after total hip arthroplasty. J Bone Joint Surg [Br] 2003;85-B:956-9.

2. Abudu A, Sivardeen KA, Grimer RJ, Pynsent PB, Noy M. The outcome of perioperative wound infection after total hip and knee arthroplasty. Int Orthop 2002;26:403

3. Fender D, Harper WM, Gregg PJ. Outcome of Charnley total hip replacement across a single health region in England: the results at five years from a regional hip register. J Bone Joint Surg [Br] 1999;81-B:577-81.

4. Garvin KL, Hanssen AD. Infection after total hip arthroplasty. J Bone Joint Surg [Am]1995;77-A:1576-86.

\section{Author's reply:}

Sir,

I thank Messrs Grimer and Abudu for the interest they have shown in our article. They are correct in saying that defining infection in total hip arthroplasty is difficult. We used a loose criterion of any wound that required extra antibiotics, in the hope that this would be very sensitive and overstate rather than understate our rate of infection. We accept that our definition was not specific and may have included false positives. As we relied on patients' recollection, we may conceivably also have had false negatives.

At present we do not have a measure that is both specific and sensitive in identifying infected arthroplasties. If it becomes mandatory for us to report infections, at surgical sites then we should report all wounds requiring extra antibiotics (very sensitive, but not specific), all positive cultures (fairly sensitive and fairly specific) and all revisions for infection (very specific, but not sensitive).

A. W. BLOM, PhD, FRCS (Tr \& Orth)

University of Bristol,

Bristol, UK.

\section{Computer-assisted knee arthroplasty versus a conventional jig-based technique}

Sir,

We read with interest the article in the April 2004 issue by Chauhan et $\mathrm{al}^{1}$ entitled 'Computer-assisted knee arthroplasty versus a conventional jig-based technique'. While we commend the authors on their study design, we would urge caution when recommending such an expensive system for routine total knee arthroplasty (TKA). The authors compared a computer-assisted knee arthroplasty with the standard protocol of intramedullary femoral and extramedullary tibial instrumentation. 
Our experience is that accurate positioning of the tibial component in coronal and sagittal planes is achieved using an intramedullary jig. ${ }^{2}$ Published studies show no difference in long-term outcome of TKA where alignment falls within $3^{\circ}$ either side of the mechanical axis. ${ }^{3-5}$ As this degree of accuracy is achievable using standard instrumentation, the benefit of navigation systems is therefore questionable in this respect. Operative time is increased significantly, particularly during the 'learning curve' period, which may affect patient outcome adversely and reduce theatre efficiency. Also, any system which uses additional ionising radiation (e.g CT scanning) introduces a small but definite cancer risk in such a large patient population.

We know that blocking the femoral and tibial canals with a bone plug prior to implantation reduces post-operative transfusion requirements. ${ }^{6}$

We disagree that computer-assisted knee arthroplasty gives better information with regard to deformity and soft-tissue balancing, as these are integral parts of the surgical procedure and dependent upon the surgeon's experience. Would computerassisted knee arthroplasty have such an effect if the surgeon had performed several thousand TKAs?

The reduction in the frequency of post-operative confusional state in the computer-assisted knee arthroplasty group is interesting. If this is indeed due to the avoidance of intramedullary instrumentation, it may be justified.

Despite our reservations, it is likely that computer-assisted knee arthroplasty in some form will become accepted practice in the future, but at present we remain unconvinced, despite the sophistication of the technology.

\section{A. J. LANGDOWN, FRCS ( $\operatorname{Tr} \&$ Orth) \\ J. AULD \\ W. J. M. BRUCE \\ Concord Repatriation Hospital, \\ Concord, New South Wales, Australia.}

1. Chauhan SK, Scott RG, BreidahI W, Becker RJ. Computer-assisted knee arthro plasty versus a conventional jig-based technique: a randomised, prospective trial. $J$ Bone Joint Surg [Br] 2004;86-B:372-7.

2. Reed MR, Bliss W, Sher JL, et al. Extramedullary or intramedullary tibial alignment guides: a randomised, prospective trial of radiological alignment. $J$ Bone Joint Surg [Br] 2002;84-B:858-60.

3. Jeffery RS, Morris RW, Denham RA. Coronal alignment after total knee replacement. J Bone Joint Surg [Br] 1991;73-B:709-14.

4. Ranawat CS, Boachie-Adjei 0. Survivorship analysis and results of total condylar knee arthroplasty: eight- to 11-year follow-up period. Clin Orthop 1988;226:6-13.

5. Bankes MJ, Back DL, Cannon SR, Briggs TW. The effect of component malalignment on the clinical and radiological outcome of the Kinemax total knee replacement. Knee 2003;10:55-60

6. Ko PS, Tio MK, Tang YK, Tsang WL, Lam JJ. Sealing the intramedullary femora canal with autologous bone plug in total knee arthroplasty. J Arthroplasty 2002;18:6-9.

\section{Author's reply}

Sirs,

I thank Messrs Langdown, Auld and Bruce for their interest in our paper. However, we do not recommend the use of computer navigation in routine knee arthroplasty as they allege. Indeed, we would urge that this new and developing technology be limited to specialised centres until such time as the technique has been adequately evaluated and its safety proven by well-designed prospective studies.

We concur with the authors' contention that a high degree of accuracy is achieved by experienced surgeons particularly if crude tools such as plain radiographs are used to assess this accuracy. However, we felt that in order to measure the accuracy of a technique which purports to be targeting accurately within $0.5^{\circ}$ and $0.5 \mathrm{~mm}$, an equally accurate measurement tool was required. This is why the CT protocol was developed ${ }^{1}$ and why plain radiographs were not chosen. This study was designed to test whether one experienced surgeon achieved greater accuracy where the only variable was chosen to be computer-assisted jigging or conventional jigging, and we have proven this.

Operative time is indeed increased by computer navigation as we state in the paper, but this will improve as the technology progresses. We would like to point out that no ionising radiation is used during knee arthroplasty using the Stryker Knee Navigation System, which is an imageless system not requiring acquisition of radiographic images such as CT scans. The CT protocol used in our study was simply a measurement tool used post-operatively. Blocking femoral and tibial canals with a bone plug is a laudable practice which we would endorse but, sadly, in our experience is infrequently done.

We did not state that computer navigation "gives better information with regard to deformity and soft-tissue balancing”. We agree that these depend upon the experience of the surgeon, and the benefit of computer navigation in this regard requires further studies.

As orthopaedic surgeons, we yearn for more accuracy and we believe our paper has demonstrated this. This improved accuracy has not yet, in short-term follow-up, delivered improvement in functional outcomes.

\section{R. J. BEAVER, FRACS \\ Royal Perth Hospital, \\ Perth, Australia.}

1. Chauhan SK, Clark GW, Lloyd S, et al. Computer-assisted total knee replacement a controlled cadaver study using a multi-parameter quantitative CT assessment of alignment (The Perth CT protocol). J Bone Joint Surg [Br] 2004;86-B:818-23.

\section{Factors affecting rates of infection and nonunion in intramedullary nailing}

Sir,

I read with interest the paper in the May 2004 issue by Malik et al ${ }^{1}$ entitled 'Factors affecting rates of infection and nonunion in intramedullary nailing'.

All efforts to identify and reduce risk factors for infection and nonunion in intramedullary nailing should be applauded, but a thorough understanding of the factors being examined is necessary for accurate interpretation of data. The authors' paper found that open fractures, fractures opened during surgery and the length of surgery were risk factors for varying degrees of infection and for nonunion. Most interesting however, is the authors use of the American Society of Anaesthesiologists (ASA) grade to calculate probabilities of nonunion.

I am sure that Saklad ${ }^{2}$ and Dripps, Lamont and Eckenhoff ${ }^{3}$ would be most upset to see that the credit for their work in developing the ASA system has been given to Sarmiento by the authors of this paper. Had the authors looked properly at the ASA system, they would realise that it is not intended for use in developing surgical outcome measures. More importantly, they would have found that the ASA system has never been validated as it has poor interobserver error with individual agreement on patients' ASA status, ranging from $13 \%$ to $85 \%$, giving a $40 \%$ to $60 \%$ chance 
that the patients' ASA grade used in their study may be inaccurate. ${ }^{4}$ Numerous other papers exist commenting on the inappropriate use of the ASA system for audit and research purposes. ${ }^{5-7}$

As such, the conclusions of their paper, of which the only new finding is a relationship between ASA grade and nonunion, must be interpreted on the understanding that there is likely to be a $40 \%$ to $60 \%$ error if applied to individual cases.

I would strongly discourage the authors from including the ASA system as an outcome measure in their ongoing randomised controlled trial.

\section{B. J. DAVIS, MRCS}

West Suffolk Hospital,

Bury St Edmunds, UK.

1. Malik MHA, Harwood P, Diggle P, Khan SA. Factors affecting rates of infection and nonunion in intramedullary nailing. J Bone Joint Surg [Br] 2004;86-B:556-60.

2. Saklad M. Grading of patients for surgical procedures. Anesthesiology 1941;2:281-4.

3. Dripps RD, Lamont A, Eckenhoff JE. The role of anesthesia in surgical mortality. JAMA 1961;178:261-6.

4. Mak PHK, Campbell RCH, Irwin MG. The ASA physical status classification: interobserver consistency. Anaesthesia and Intensive Care 2002;30:633-40.

5. Owens WD, Felts JA, Spitznagel EJ. ASA physical status classifications. Anesthesiology 1978;49:239-43.
6. Haynes SR, Lawler PGP. An assessment of the consistency of ASA physical status classification allocation. Anaesthesia 1995;50:195-9.

7. Ranta S, Hynynen M, Tammisto T. A survey of the ASA physical status classification: significant variation in allocation among Finnish anaesthesiologists. Acta Anaesthesiol Scand 1997;41:629-32.

\section{Author's reply}

Sir,

We thank Mr Davis for the interest he has shown in our paper. Although there may be interobserver variation in the allocation of the American Society of Anaesthesiologists score, it still remains the most widely used and accepted method of physical status classification and as such has gained worldwide acceptance. Our paper demonstrates that it offers an easily accessible means of identifying patients who may be at increased risk of developing nonunion following intramedullary nail insertion and be of use in modifying clinical practice. We await the results of our prospective study to show whether or not this is the case.

\section{H. A. MALIK}

Wrightington Hospital, Wigan, UK. 